\title{
Erratum: Detection of Macroscopic Entanglement by Correlation of Local Observables [Phys. Rev. Lett. 95, 090401 (2005)]
}

\author{
Akira Shimizu and Tomoyuki Morimae \\ (Received 20 October 2016; published 16 November 2016)
}

DOI: 10.1103/PhysRevLett.117.219903

Although the index $q$ was proposed for mixed states, it is also applicable to pure states, on which we correct the following statements.

Page 2, left column, 4th last line: " $q=1$ " should read " $q<2$."

Page 3, left column, paragraph just after Eq. (6): "Since addition of ... entanglement [5,6], $p=1$ for $|\phi\rangle$ if $p=1$ for $|\psi\rangle$. Thus, from inequality (6), we find that if $p=1$ then $q=1$. In particular, $q=1$ for any product state $|\psi\rangle=\bigotimes_{l=1}^{N}\left|\psi_{l}\right\rangle$ because $p=1$. To sum up, we have found that $p=1 \Leftrightarrow q=1$ and that ..."

should read

"For any product state $|\psi\rangle=\bigotimes_{l=1}^{N}\left|\psi_{l}\right\rangle$, addition of ... entanglement [5,6]. Thus, from inequality (6), we find that $q=1$ for any product state. To sum up, we have found that $q=1 \Rightarrow p=1, p=1 \Rightarrow q \leq 1.5$ and that ...."

Page 4, right column, line 3: "which has $q=1$ " should read "which has $q=1.5$."

Furthermore, for clarity, we also make the following improvements:

Page 2, left column, 2nd paragraph: Insert "with nonvanishing errors" after "if one looks only at such expectation values". Page 3, left column, three lines after Eq. (6): Insert "and that if $p=1$ then $q \leq 1.5$ " after "if $q=2$ then $p=2$ ". We remark that the conclusions of the present work remain unchanged.

We thank Y. Matsuzaki for discussions. 